\title{
Nanoparticle Interactions and Molecular Relaxation in PLA/PBAT/Nanoclay Blends
}

\author{
M. Nofar ${ }^{1, *}$ (D), M.-C. Heuzey ${ }^{2}$, P.J. Carreau ${ }^{2}$ and M.R. Kamal ${ }^{3}$ \\ ${ }^{1}$ Department of Metallurgical and Materials Engineering, Faculty of Chemical and Metallurgical Engineering, Istanbul \\ Technical University, 34469 Maslak, Istanbul, Turkey, ${ }^{2}$ Center for High Performance Polymer and Composite systems \\ (CREPEC), Chemical Engineering Department, Polytechnique Montreal, Montreal, Quebec H3T 1J4, Canada, and ${ }^{3}$ CREPEC, \\ Chemical Engineering Department, McGill University, Montreal, Quebec H3A 2B2, Canada \\ *Author to whom correspondence should be addressed: nofar@itu.edu.tr
}

(Received 20 July 2020; Revised 13 September 2020; Accepted 21 September 2020)

\begin{abstract}
Organo-modified clay nanoparticles were mixed at 1 and $5 \mathrm{wt} \%$ concentrations with a molten blend of $75 \mathrm{wt} \%$ of polylactide (PLA) and $25 \mathrm{wt} \%$ poly[(butylene adipate)-co-terephthalate] (PBAT). Three mixing strategies were used to control the localization of nanoclay. Small amplitude oscillatory shear (SAOS) and stress growth tests were conducted to clarify the nanoclay interactions with the blend components and its effect on the molecular relaxation behavior. SAOS and weighted relaxation spectra properties were determined before and after pre-shearing at a rate of $0.01 \mathrm{~s}^{-1}$. Molecular relaxation and its characteristics were influenced by PLA degradation, PBAT droplet coalescence, and nanoclay localization.
\end{abstract}

Keywords: PLA; Blend; Rheology

\section{Introduction}

Polymer blending is used to obtain tailored combinations of properties (Favis, 1991; Macosko, 2000; Nofar et al., 2019b). The morphological characteristics of a binary polymer blend are determined by thermodynamics, processing conditions, type of dispersed phase, and viscoelastic behavior of the polymers under melt blending conditions (Favis \& Chalifoux, 1987; Favis \& Therrien, 1991; Nofar et al., 2016; 2017; 2019a; 2019b; Scott \& Macosko, 1991; Souza \& Demarquette, 2002; Sundararaj et al., 1995). Blend nanocomposites are systems that exhibit variety of microstructures (Dil \& Favis, 2015; Maani \& Carreau, 2016; Nofar et al., n.d.). The interfacial interactions, rheological properties and the processing conditions determine the localization of the nanoparticles and influence the final properties (Maani \& Carreau, 2016; Nofar et al., 2019b; n.d.; Sumita et al., 1991).

Blending with other flexible biopolymers and the incorporation of nanoparticles has been shown to be a promising approach to overcome several drawbacks of polylactide (PLA) (Keshtkar et al., 2014; Nofar, 2016; 2018; Nofar \& Park, 2014; 2015; 2017; Nofar et al., 2013; 2019b; Raquez et al., 2013). Few researchers have studied the processing-morphology-property relationships in nanocomposites based on PLA/poly[(butylene adipate)-co-terephthalate] (PBAT) blends (Dil \& Favis, 2015; Dil et al., 2016; Jiang et al., 2009; Ko et al., 2009; Kumar et al., 2010; Li et al., 2011; Nofar et al., 2019b; n.d.; Shi et al., 2016). Jiang et al. (2009) showed when using a twin-screw extruder, the montmorillonite clay was dispersed

\footnotetext{
(C) The Author(s), 2020. Published by Cambridge University Press. This is an Open Access article, distributed under the terms of the Creative Commons Attribution licence (http://creativecommons.org/licenses/by/4.0/), which permits unrestricted re-use, distribution, and reproduction in any medium, provided the original work is properly cited.
} 
throughout the whole blend. Kumar et al. (2010) reported a similar localization for Cloisite 20A. Nofar et al. (n.d.) showed that the Cloisite 30B nanoclay was localized at the interface between PLA and PBAT phases and acted as a barrier to droplet coalescence. In all of these studies, the rheological properties are critical parameters in determining the blend morphology and nanoparticle localization (Nofar et al., 2019b; 2019c). In this study, in complement to the results of Nofar et al. (n.d.), we present additional SAOS and weighted relaxation spectra data to analyze the nanoparticle interactions with the blend components and the effect of pre-shearing. Investigating the effect of shearing is important since during the melt processing, the applied shear flow could influence the morphology and the properties of a system.

\section{Experimental}

\section{2-1- Materials and Sample Preparation}

A commercial amorphous linear PLA, Ingeo 4060D, PBAT, Ecoflex ${ }^{\odot} \mathrm{F}$ Blend C1200, (Dil et al., 2015) and organo-modified nanoclay Cloisite 30B (C30B) were used in this study (Krikorian \& Pochan, 2003). The weight-average molecular weight of these PLA and PBAT samples were 180 and $126 \mathrm{~kg} / \mathrm{mol}$, respectively. The PBAT possessed around 15\% of crystallinity.

Blend nanocomposites were prepared containing $25 \mathrm{wt} \%$ PBAT and 1 or $5 \mathrm{wt} \% \mathrm{C} 30 \mathrm{~B}$ based on the total weight. The compounding was conducted using an internal batch mixer, DDRV501 Brabender at $100 \mathrm{rpm}$ and $160^{\circ} \mathrm{C}$ for $12 \mathrm{~min}$. Three different strategies were employed for preparing the nanocomposites which are reported in (Nofar et al., n.d.) and are referred to as S1, S2, and S3. Disk shape rheological specimens were pressed at $160^{\circ} \mathrm{C}$.

\section{2-2- Rheological Measurements}

Rheological measurements were conducted at $160^{\circ} \mathrm{C}$ under a nitrogen atmosphere using an Anton Paar MCR-301 rotational rheometer. The thermal stability and the viscoelastic properties of the neat polymers and their blend nanocomposites were examined using time and frequency sweep tests. Stress-growth experiments were carried out at a shear rate of $0.01 \mathrm{~s}^{-1}$. This low shear rate was selected to investigate droplet coalescence in the blends in absence of break up (Nofar et al., 2015). In order to investigate the effect of morphological changes during shearing on the rheological behavior of the blends, SAOS tests were also performed after stress growth and relaxation of the samples.

\section{Results and Discussion}

\section{3-1- Thermal Stability}

Figure 1 reports the viscosity variations with time of the neat PLA, PLA-PBAT blend and the PLA-PBAT blend nanocomposites containing 1 and $5 \mathrm{wt} \% \mathrm{C} 30 \mathrm{~B}$. It was depicted that $\mathrm{C} 30 \mathrm{~B}$ expedited the degradation of PLA (Najafi et al., 2012; Nofar et al., n.d.). As Figure 1 shows, after 910 s the PLA viscosity decreases by about $10 \%$, whereas for the PLA-PBAT blend, the $10 \%$ viscosity drop is reached after only $500 \mathrm{~s}$ due to the additional effect of PBAT droplet coalescence. In blend nanocomposites with $1 \mathrm{wt} \% \mathrm{C} 30 \mathrm{~B}$, the $10 \%$ viscosity drop occurs after 1,080, 1,010, and 1,180 s in samples prepared through S1, S2, and S3, respectively. As shown in morphological analysis from our previous study (Nofar et al., n.d.), the localization of clay at the interface eliminates or seriously reduces the droplet coalescence. Further, the viscosity reduction is more important in the order of S2 $>\mathrm{S} 1>\mathrm{S} 3$. This is more obvious in the blend nanocomposites containing $5 \mathrm{wt} \%$ clay prepared through S1, S2, and S3, as the $10 \%$ viscosity drop occurs after 1,130,930, and 2,230 s, respectively. Obviously, the stability is significantly better for $\mathrm{S} 3$ than for the other two samples. This is because the PLA degradation in the presence of nanoclay was minimized as the clay was initially mixed with PBAT and subsequently migrated to the interface (Nofar et al., n.d.). 


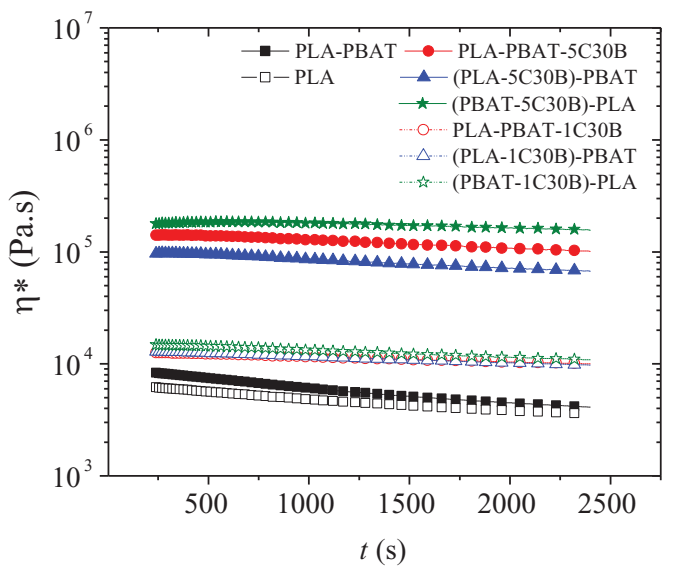

Figure 1. Complex viscosity as a function of time at $160^{\circ} \mathrm{C}$
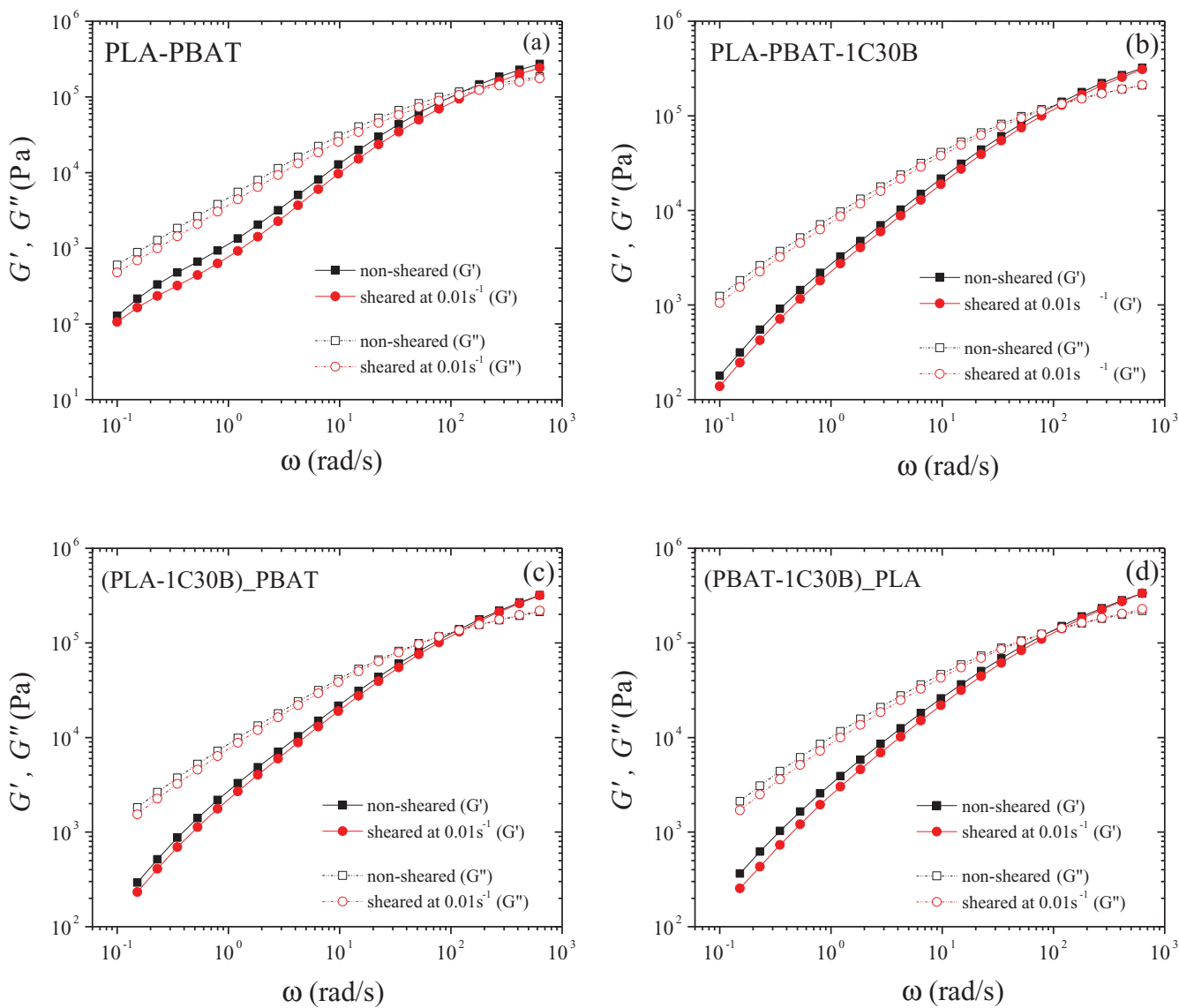

Figure 2. Moduli of non-sheared/sheared (a) PLA-PBAT and (b-d) nanocomposites prepared via (b) S1, (c) S2, (d) S3.

\section{3-2- Rheological Behavior of PLA/PBAT/C30B Systems Before and After Shearing}

Figures 2a-d present the $G$ ' and $G$ " data of the PLA-PBAT blend and its corresponding nanocomposites with $1 \mathrm{wt} \%$ clay before and after shearing at $0.01 \mathrm{~s}^{-1}$. These figures show that both moduli are 
significantly reduced by shearing mainly due to coalescence as demonstrated in our previous publication (Nofar et al., n.d.). The inverse of the $G^{\prime}$ and $G$ " crossover frequency at high frequencies is a characteristic relaxation time of the PLA chains in all systems. For PLA-PBAT blend, it is $0.0073 \mathrm{~s}$ in the non-sheared sample that reduces to $0.0059 \mathrm{~s}$ in sheared sample at $0.01 \mathrm{~s}^{-1}$. This shortened relaxation time can be attributed to the PLA molecular degradation. According to Figures $2 \mathrm{~b}-\mathrm{d}$, the addition of $1 \mathrm{wt} \%$ clay does not affect significantly the molecular relaxation time. Compared to $0.0073 \mathrm{~s}$ for the neat blend, the characteristic time is about $0.010 \mathrm{~s}$ for all blend nanocomposites independently of the preparation method and shearing. This slight increase is most likely due to the hindering effect of the nanoclay located partly in the matrix (Nofar et al., n.d.). The corresponding complex viscosity results and the related discussions are provided in the previous study (Nofar et al., n.d.).

Figures 3a-c report the G' and G" data of PLA-PBAT blend nanocomposites with $5 \mathrm{wt} \%$ clay before and after shearing at $0.01 \mathrm{~s}^{-1}$. In the non-sheared samples, the relaxation time determined for the crossover frequency increases when $5 \mathrm{wt} \%$ clay is added and, depending on the preparation strategy, this increase is in the order of $\mathrm{S} 1<\mathrm{S} 2<\mathrm{S} 3$. For example, the relaxation time for (PBAT-5C30B)-PLA is $0.025 \mathrm{~s}$ compared to about $0.010 \mathrm{~s}$ observed for (PBAT-1C30B)-PLA. This significant increase is attributed to the hindering effect of the nanoparticles that had migrated to the matrix phase under the mixing route S3 (Nofar et al., n.d.). The longest relaxation time is observed in the (PBAT-5C30B)-PLA (S3) sample compared to the S1 an S2 cases, due to the reduced PLA degradation as the PLA/clay interactions were minimized when S3 was used as the preparation method. The relaxation time values before and after shearing are as follows: (a) in S1: from 0.0127 to $0.0076 \mathrm{~s}$, (b) in S2: from 0.0167 to $0.0083 \mathrm{~s}$, and (c) in S3: from 0.0250 to $0.0118 \mathrm{~s}$, respectively. In all cases, when shearing is applied, the relaxation time decreases due to the more severe degradation of the PLA molecules in presence of $5 \mathrm{wt} \%$ clay.

Using the SAOS data, the weighted relaxation spectra of the blends were calculated (Stadler \& Bailly, 2009; Sherrod, 2010). In Figure 4, the PLA/PBAT blend reveals two relaxation peaks, where the first peak corresponds to the main relaxation time of the chains of the matrix $(0.05 \mathrm{~s})$, which is largely different from that obtained from the crossover frequency $(0.0073 \mathrm{~s})$; the second peak characterizes the interfacial relaxation between the two phases $(\sim 5 \mathrm{~s})$. After $0.01 \mathrm{~s}^{-1}$ shearing, in PLA-PBAT neat blends, the interfacial relaxation time increases from 5 to around $9 \mathrm{~s}$ due to the coalescence of PBAT droplets (Nofar et al., n.d.) and the PLA chain molecular relaxation decreases from 0.05 to around $0.04 \mathrm{~s}$, which is a consequence of molecular degradation of PLA. In PLA/PBAT/1C30B systems regardless of the preparation strategy and shearing, wide relaxation merged peaks are observed although for the samples prepared via S1 and S2 two shoulders are also seen within the wide peaks. This is probably because the molecular and shape relaxations occur at a very close range when nanoclay is mainly located at the interface. Another interesting behavior is seen in PLA-PBAT blends containing $5 \mathrm{wt} \%$ clay regardless of the preparation strategy (Figures $4 \mathrm{c}$-d). In all these cases, before and after shearing, slight shoulders at short relaxation times are observed followed by unbounded tails at longer times instead of shape relaxation peaks. These tails could be associated with the unfinished relaxation of the continuous solid network structures within the measuring frequency ranges (Kamal \& Khoshkava, 2015).
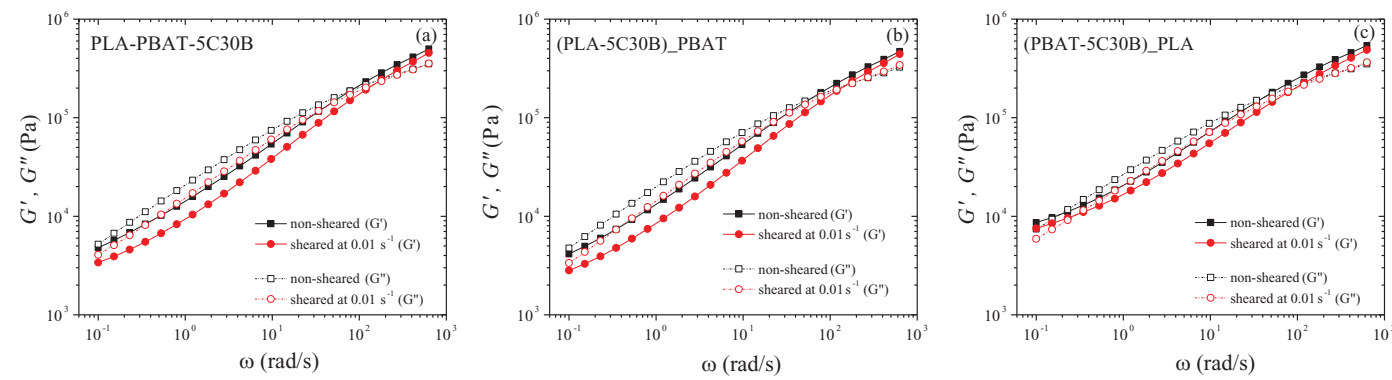

Figure 3. Moduli of non-sheared and sheared nanocomposites with 5 wt $\%$ clay prepared via (a) S1, (b) S2, and (c) S3. 

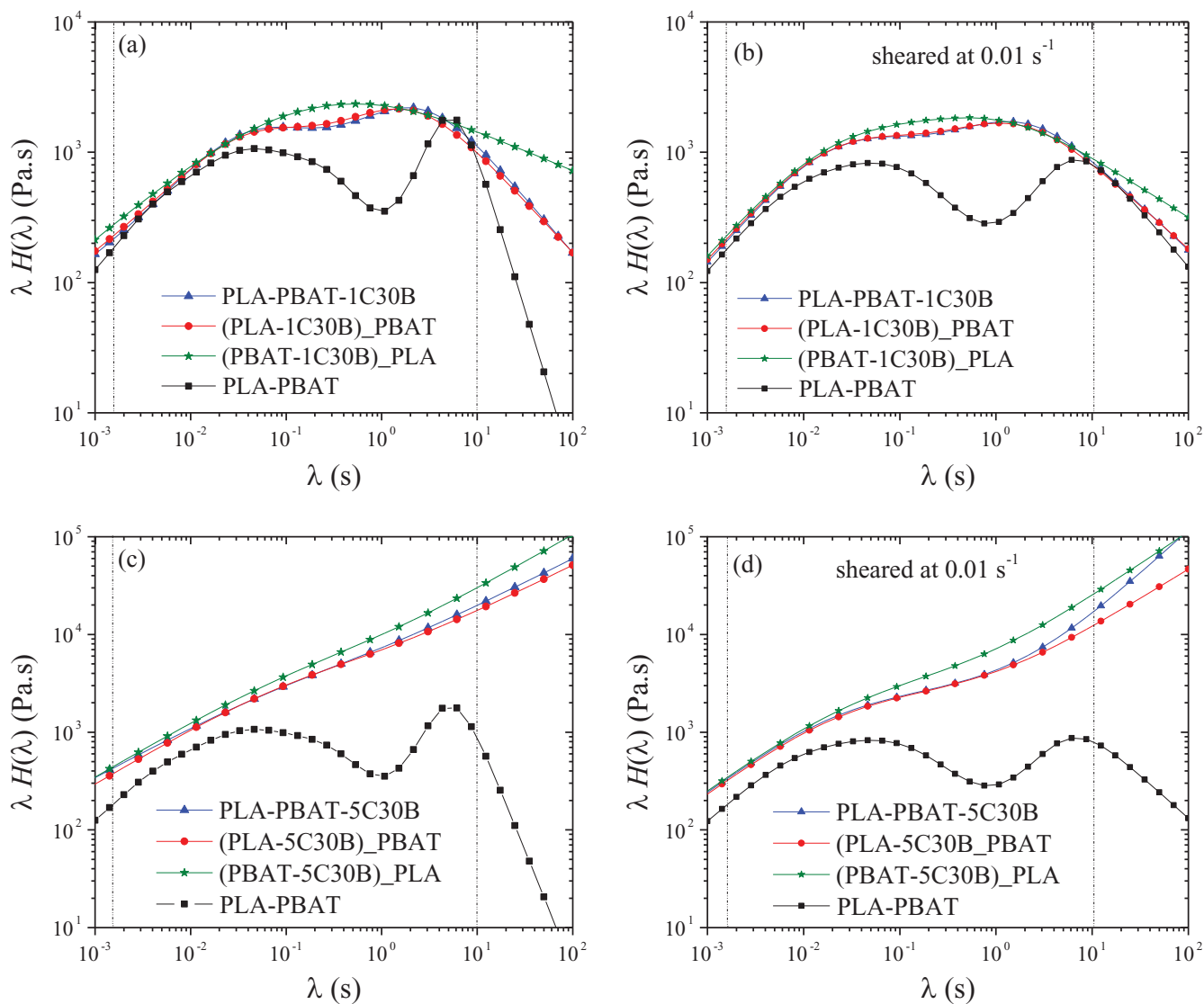

Figure 4. Weighted relaxation spectra of non-sheared (a, c) and sheared at $0.01 \mathrm{~s}^{-1}$ (b, d) PLA-PBAT blend and its nanocomposites with (a, b) 1 wt $\%$ and (c, d) 5 wt $\%$ clay.

\section{Conclusion}

In this study, 1 and $5 \mathrm{wt} \%$ nanoclay was incorporated within PLA/PBAT blends prepared via three different melt mixing strategies to investigate nanoclay interactions with the blend components and their effect on the molecular relaxation of the PLA chains. High content of nanoclay increased the relaxation time of the PLA molecules, but also contributed to a more severe PLA degradation depending on the mixing strategy. Shearing also led to more PLA degradation and decreased the relaxation time of the PLA chains. Blend preparation strategy and hence the nanoparticles localization could influence the molecular relaxation of PLA chains differently. Weighted relaxation spectra data of blends with $5 \mathrm{wt} \%$ clay exhibited a tail at long relaxation times, which could be related to the unfinished relaxation of the continuous solid network structures.

Acknowledgements. The authors would like to thank Jed Randall from NatureWorks for providing the PLA sample.

Author Contributions. M. Nofar conceived and designed the study, conducted experiments, and wrote the article. M.C Heuzey, P. Carreau and M. Kamal conceived the study and were involved in data analysis and writing the article.

Funding Information. This research received no specific grant from any funding agency, commercial or not-for-profit sectors.

Conflict of Interest. Authors declare none.

Data availability. To view supplementary material for this article, please visit https://doi.org/10.1016/j.polymer.2016.06.044. 


\section{References}

Dil, E. J., Carreau, P. J., \& Favis, B. D. (2015). Morphology, miscibility and continuity development in poly (lactic acid)/poly (butylene adipate-co-terephthalate) blends. Polymer, 68, 202-212.

Dil, E. J., \& Favis, B. D. (2015). Localization of micro-and nano-silica particles in heterophase poly (lactic acid)/poly (butylene adipate-co-terephthalate) blends. Polymer, 76, 295-306.

Dil, E. J., Virgilio, N., \& Favis, B. D. (2016). The effect of the interfacial assembly of nano-silica in poly (lactic acid)/poly (butylene adipate-co-terephthalate) blends on morphology, rheology and mechanical properties. Euroean Polymer Journal, 85, 635-646.

Favis, B. D. (1991). Polymer alloys and blends: Recent advances. The Canadian Journal of Chemical Engineering, 69, 619-625.

Favis, B. D., \& Chalifoux, J. P. (1987). The effect of viscosity ratio on the morphology of polypropylene/polycarbonate blends during processing. Polymer Engineering \& Science, 27, 1591-1600.

Favis, B. D., \& Therrien, D. (1991). Factors influencing structure formation and phase size in an immiscible polymer blend of polycarbonate and polypropylene prepared by twin-screw extrusion. Polymer, 32, 1474-1481.

Jiang, L., Liu, B., \& Zhang, J. (2009). Properties of poly (lactic acid)/poly (butylene adipate-co-terephthalate)/nanoparticle ternary composites. Industrial \& Engineering Chemistry Research, 48, 7594-7602.

Kamal, M. R., \& Khoshkava, V. (2015). Effect of cellulose nanocrystals (CNC) on rheological and mechanical properties and crystallization behavior of PLA/CNC nanocomposites. Carbohydrate Polymers, 123, 105-114.

Keshtkar, M., Nofar, M., Park, C. B., \& Carreau, P. (2014). Extruded PLA/clay nanocomposite foams blown with supercritical $\mathrm{CO}^{2}$. Polymer, 55, 4077-4090.

Ko, S. W., Hong, K. M., Park, B. J., Gupta, K. R., Choi, H. J., \& Bhattacharya, S. N. (2009). Morphological and rheological characterization of multi-walled carbon nanotube/PLA/PBAT blend nanocomposites. Polymer Bulletin, 63, $125-134$.

Krikorian, V., \& Pochan, D. J. (2003). Poly (l-lactic acid)/layered silicate nanocomposite: fabrication, characterization, and properties. Chemistry of Materials, 15, 4317-4324.

Kumar, M., Mohanty, S., Nayak, S., \& Parvaiz, M. R. (2010). Effect of glycidyl methacrylate (GMA) on the thermal, mechanical and morphological property of biodegradable PLA/PBAT blend and its nanocomposites. Bioresource Technology, 101, 8406-8415.

Li, K., Cui, Z., Sun, X., Turng, L.-S., \& Huang, H. (2011). Effects of nanoclay on the morphology and physical properties of solid and microcellular injection molded polyactide/poly(butylenes adipate-co-terephthalate) (PLA/PBAT) nanocomposites and blends. Journal of Biobased Materials and Bioenergy, 5, 442-451.

Maani, A., \& Carreau, P. J. (2016). Rheological and morphological properties of thermoplastic olefin blends containing nanosilica. Journal of Non-Newtonian Fluid Mechanics, 233, 95-106.

Macosko, C. W. (2000). Morphology development and control in immiscible polymer blends. Macromolecular Symposia, 149, 171-184.

Najafi, N., Heuzey, M.-C., Carreau, P. J., \& Wood-Adams, P. M. (2012). Control of thermal degradation of polylactide (PLA)clay nanocomposites using chain extenders. Polymer Degradation and Stability, 97, 554-565.

Nofar, M. (2016). Effects of nano-/micro-sized additives and the corresponding induced crystallinity on the extrusion foaming behavior of PLA using supercritical CO². Materials \& Design, 101, 24-34.

Nofar, M. (2018). Rheological, thermal, and foaming behaviors of different polylactide grades. International Journal of Material Science and Research, 1, 16-22. https://doi.org/10.18689/ijmsr-1000103.

Nofar, M., Heuzey, M.-C., Carreau, P., \& Kamal, M. (2016). Effects of nanoclay and its localization on the morphology stabilization of PLA/PBAT blends under shear flow. Polymer, 98, 353-364.

Nofar, M., Heuzey, M. C., Carreau, P. J., Kamal, M. R., \& Randall, J. (2016). Coalescence in PLA-PBAT blends under shear flow: Effects of blend preparation and PLA molecular weight. Journal of Rheology, 60, 637-648.

Nofar, M., Maani, A., Sojoudi, H., Heuzey, M. C., \& Carreau, P. J. (2015). Interfacial and rheological properties of PLA/PBAT and PLA/PBSA blends and their morphological stability under shear flow. Journal of Rheology, 59, 317-333.

Nofar, M., Oguz, H., \& Ovali, D. (2019a). Effects of the matrix crystallinity, dispersed phase, and processing type on the morphological, thermal, and mechanical properties of polylactide-based binary blends with poly[(butylene adipate)-coterephthalate] and poly[(butylene succinate)-co-adipate]. Journal of Applied Polymer Science, 136, 47636-47647.

Nofar, M., \& Park, C. B. (2014). Poly (lactic acid) foaming. Progress in Polymer Science, 39, 1721-1741.

Nofar, M., \& Park, C. B. (2015). Chapter 5 - Heterogeneous cell nucleation mechanisms in polylactide foaming. In: S. Iannace \& C. B.Park (Eds.), Biofoams: Science and applications of bio-based cellular and porous materials (pp. 153-177). CRC Press, ISBN-13: 978-1-4665-6180-9.

Nofar, M., \& Park, C. B. (2017). Polylactide foams: Fundamentals, manufacturing, and applications. Elsevier, William Andrew, ISBN: 9780128139912.

Nofar, M., Sacligil, D., Carreau, P. J., Kamal, M. R., \& Heuzey, M. C. (2019b). Poly (lactic acid) blends: Processing, properties and applications. International Journal of Biological Macromolecules, 125, 307-360.

Nofar, M., Salehiyan, R., \& Ray, S. S. (2019c). Rheology of poly (lactic acid)-based systems. Polymer Reviews, 59, 465-509.

Nofar, M., Tabatabaei, A., \& Park, C. B. (2013). Effects of nano-/micro-sized additives on the crystallization behaviors of PLA and PLA/CO ${ }^{2}$ mixtures. Polymer, 54, 2382-2391. 
Nofar, M., Tabatabaei, A., Sojoudiasli, H., Park, C., Carreau, P., Heuzey, M.-C., \& Kamal, M. (2017). Mechanical and bead foaming behavior of PLA-PBAT and PLA-PBSA blends with different morphologies. European Polymer Journal, 90, 231-244.

Raquez, J.-M., Habibi, Y., Murariu, M., \& Dubois, P. (2013). Polylactide (PLA)-based nanocomposites. Progress in Polymer Science, 38, 1504-1542.

Scott, C. E., \& Macosko, C. W. (1991). Model experiments concerning morphology development during the initial stages of polymer blending. Polymer Bulltin, 26, 341-348.

Sherrod, P. H. (2010). Nonlinear regression analysis program. http://www.nlreg.com

Shi, X., Zhang, G., Liu, Y., Ma, Z., Jing, Z., \& Fan, X. (2016). Microcellular foaming of polylactide and poly (butylene adipateco-terphathalate) blends and their $\mathrm{CaCO}_{3}$ reinforced nanocomposites using supercritical carbon dioxide. Polymers for Advanced Technologies, 27, 550-560.

Souza, A., \& Demarquette, N. (2002). Influence of coalescence and interfacial tension on the morphology of PP/HDPE compatibilized blends. Polymer, 43, 3959-3967.

Stadler, F. J., \& Bailly, C. (2009). A new method for the calculation of continuous relaxation spectra from dynamic-mechanical data. Rheologica Acta, 48, 33-49.

Sumita, M., Sakata, K., Asai, S., Miyasaka, K., \& Nakagawa, H. (1991). Dispersion of fillers and the electrical conductivity of polymer blends filled with carbon black. Polymer Bulletin, 25, 265-271.

Sundararaj, U., Macosko, C. W., Nakayama, A., \& Inoue, T. (1995). Milligrams to kilograms: An evaluation of mixers for reactive polymer blending. Polymer Engineering \& Science, 35, 100-114.

Cite this article: Nofar M, Heuzey M-C, Carreau PJ, Kamal MR (2020). Nanoparticle Interactions and Molecular Relaxation in PLA/PBAT/Nanoclay Blends Experimental Results, 1, e47, 1-12. https://doi.org/10.1017/exp.2020.54 


\section{Peer Reviews}

\section{Reviewing editor: Prof. Maria Norberta De Pinho}

Universidade de Lisboa Instituto Superior Tecnico, Lisboa, Portugal, 1049-001

This article has been accepted because it is deemed to be scientifically sound, has the correct controls, has appropriate methodology and is statistically valid, and has been sent for additional statistical evaluation and met required revisions.

doi:10.1017/exp.2020.54.pr1

Review 1: Nanoparticle Interactions and Molecular Relaxation in PLA/PBAT/Nanoclay Blends

Reviewer: Prof. Maria Helena Godinho

Date of review: 28 July 2020

(C) The Author(s), 2020. Published by Cambridge University Press This is an Open Access article, distributed under the terms of the Creative Commons Attribution licence (http://creativecommons.org/licenses/by/4.0/), which permits unrestricted re-use, distribution, and reproduction in any medium, provided the original work is properly cited.

Conflict of interest statement. Reviewer declares none

Comments to the Author: The work presented in this manuscript complements the results published previously by the authors, namely in 2016 (ref. [13]). Further rheological studies are presented by the authors concerning two systems prepared from a molten blend of polylactide (PLA) and poly[(butylene adipate)-co-terephthalate] (PBAT) mixed with 1 and $5 \mathrm{wt} \%$ of clay nano particles.

While in previous works the authors used different techniques, including morphological studies, to characterize the mixtures prepared the present work is dedicated only to rheological measurements. To understand the rheological results obtained and the analyses performed, in this work, the previous results published by the authors should be accessed.

In this manuscript the authors concluded that PLA behavior and characteristics are influenced not only by the presence of the nanoparticles but also by sample preparation and shearing conditions. Two main points stressed by the authors should be deeply addressed and explained "PLA degradation" and "unfinished relaxation of the continuous solid..." in light of the results presented in this manuscript and previously reported by the authors.

\section{Score Card}

Presentation 
Does the abstract correctly embody the content of the article? (25\%)

Does the introduction give appropriate context? (25\%)

Is the objective of the experiment clearly defined? (25\%)

Analysis

Does the discussion adequately interpret the results presented? (40\%)

Is the conclusion consistent with the results and discussion? (40\%)

Are the limitations of the experiment as well as the contributions of the experiment clearly outlined? $(20 \%)$ 


\section{Review 2: Nanoparticle Interactions and Molecular Relaxation in PLA/PBAT/Nanoclay Blends}

Reviewer: Dr. Reza Salehiyan

Council for Scientific and Industrial Research, South Africa

Date of review: 08 September 2020

(C) The Author(s), 2020. Published by Cambridge University Press This is an Open Access article, distributed under the terms of the Creative Commons Attribution licence (http://creativecommons.org/licenses/by/4.0/), which permits unrestricted re-use, distribution, and reproduction in any medium, provided the original work is properly cited.

Conflict of interest statement. I declare no conflict of interest with this manuscript.

Comments to the Author: The manuscript describes an interesting subject. The effect of C30B clay and its sequence of addition to the PLA/PBAT on the rheological properties and relaxation spectra are discussed. The discussions are in agreement with the results. The manuscript is written in such a way that meets the standards to be published. The results are adequate and the manuscript is well-structured. I vote for the acceptance of this manuscript for publication.

\section{Score Card}

Presentation

5.0

Does the paper cite relevant and related articles appropriately? (30\%)
Context

5.0

Does the title suitably represent the article? (25\%)

Does the abstract correctly embody the content of the article? (25\%)

Does the introduction give appropriate context? (25\%)

Is the objective of the experiment clearly defined? $(25 \%)$

Analysis

5.0
Does the discussion adequately interpret the results presented? (40\%)

Is the conclusion consistent with the results and discussion? (40\%)

Are the limitations of the experiment as well as the contributions of the experiment clearly outlined? $(20 \%)$ 


\section{Review 3: Nanoparticle Interactions and Molecular Relaxation in PLA/PBAT/Nanoclay Blends}

Reviewer: Dr. Amirjalal Jalali iD

University of Toronto, MIE, Canada

Date of review: 05 September 2020

(C) The Author(s), 2020. Published by Cambridge University Press This is an Open Access article, distributed under the terms of the Creative Commons Attribution licence (http://creativecommons.org/licenses/by/4.0/), which permits unrestricted re-use, distribution, and reproduction in any medium, provided the original work is properly cited.

Conflict of interest statement. Reviewer declares none

Comments to the Author: The manuscript "Nanoparticle Interactions and Molecular Relaxation in PLA/PBAT/Nanoclay Blends" written by Nofar et al. employs rotational rheometry measurements to determine the localization of organoclay additive in a PLA/PBAT blend (75/25 wt $\%)$ containing different concentration of nanofiller. This paper is written in really good English and precisely investigates the rheological behavior of the nanocomposites and relaxation of the polymer chains by means of rheological analysis. This article for sure deserves publication and, to me, it is accepted to be published in Experimental Results Journal; however, it needs the following minor corrections as follows:

1. What is the crystalinity amount of PLA and PBAT? Also please mention the molecular weight of the applied polymeric materials in this study, if possible.

2. The authors mention "The inverse of the G' and G" crossover frequency at high frequencies is a characteristic relaxation time of the PLA chains in all systems". There are different ways in literature to calculate and analyze the relaxation time. Please mention the other methods and explain why you selected this way (using distribution of relaxation time)?

3. The authors discuss about the role of shearing. Please explain why you are willing to investigate the effect of shearing on nanocomposites.

4. The variation of G' and G" was studied and results are presented. I would like to know how the complex viscosity varies by addition of nano-filler and shearing. Please explain the general trend in some sentences, if possible.

\section{Score Card}

Presentation

5.0

Is the article written in clear and proper English? (30\%)

Is the data presented in the most useful manner? (40\%)

Does the paper cite relevant and related articles appropriately? (30\%)

Context

Does the title suitably represent the article? (25\%)

Does the abstract correctly embody the content of the article? (25\%)

Does the introduction give appropriate context? (25\%) 
Is the objective of the experiment clearly defined? (25\%)

Analysis

Does the discussion adequately interpret the results presented? (40\%)

Is the conclusion consistent with the results and discussion? (40\%)

Are the limitations of the experiment as well as the contributions of the experiment clearly outlined? $(20 \%)$ 